Relations industrielles

Industrial Relations

\title{
Le colloque CTC-PSD à Winnipeg
}

\section{Fernand Bourret}

Volume 14, numéro 4, octobre 1959

URI : https://id.erudit.org/iderudit/1022131ar

DOI : https://doi.org/10.7202/1022131ar

Aller au sommaire du numéro

Éditeur(s)

Département des relations industrielles de l’Université Laval

ISSN

0034-379X (imprimé)

1703-8138 (numérique)

Découvrir la revue

Citer ce document

Bourret, F. (1959). Le colloque CTC-PSD à Winnipeg. Relations industrielles / Industrial Relations, 14(4), 573-589. https://doi.org/10.7202/1022131ar
Résumé de l'article

L'action politique préoccupe le mouvement ouvrier. A la fin d'août 1959, se réunissaient à Winnipeg des représentants du parti social démocratique et du Congrès du travail du Canada dans le but d'étudier la possibilité de créer en notre pays un nouveau parti politique. Voici un reportage objectif de ce qui s'est passé avec le texte même des déclarations importantes.
Tous droits réservés @ C Département des relations industrielles de l’Université Laval, 1959
Ce document est protégé par la loi sur le droit d'auteur. L’utilisation des services d'Érudit (y compris la reproduction) est assujettie à sa politique d'utilisation que vous pouvez consulter en ligne.

https://apropos.erudit.org/fr/usagers/politique-dutilisation/ 


\section{COMMENTAIRES}

Le colloque CTC-PSD à Winnipeg

\section{Fernand Bourret}

L'action politique préoccupe le mouvement ouvrier. A la fin daoût 1959, se réunissaient d̀ Winnipeg des représentants du parti social démocratique et du Congrès du travail du Canada dans le but détudier la possibilité de créer en notre pays un nouveau parti politique. Voici un reportage objectif de ce qui s'est passé avec le texte même des déclarations importantes.

* Je sais que je parle au nom des quelque 300 délégués qui ont participé à ce séminar national quand je déclare qu'il a remporté un grand suocès ». C'est ainsi que s'exprimait M. Stanley Knowles, président du comité conjoint CTC-PSD, lors de la séance de clôture du colloque des deux groupes à Winnipeg, les 28, 29 et 30 août dernier.

«Ce séminar, a-t-il ajouté, a fourni aux délégués des différents groupes représentés, l'occasion de discuter abondamment et franchement. Ces discussions ont été précieuses car elles ont permis de faire l'unanimité sur les principales questions.

«En vérité, dit-il, l'unité que nous avons réussi à faire constitue l'une des principales caractéristiques de notre rencontre, malgré les tentatives de la presse et de certains groupes pour nous diviser ».

En quelques mots, sommairement, le président du comité conjoint CTC-PSD venait de résumer les résultats obtenus après trois journées de travail ardu des 300 délégués en provenance de toutes les parties du Canada et qui représentaient des syndicats de travailleurs, de toutes les provinces canadiennes, des fermiers des provinces de l'Ouest et de l'Ontario, des intellectuels et des universitaires, des représentants du parti CCF national et des différentes sections provinciales.

«Le programme que nous nous étions tracé, a continué M. Knowles, devait se réaliser en deux étapes. La première étape consistait à obtenir des suggestions des membres du parti CCF et du Congrès du travail du Canada; la seconde devait placer devant eux des suggestions concrètes pour l'élaboration d'un programme et d'une constitution à préciser plus tard.

«Ce seminar, a-t-il dit, était la première phase de la deuxième étape ». 
LA RÉSOLUTION DE WINNIPEG

Voici étape par étape comment s'est déroulé le processus permettant la rencontre, à ce stage, des dirigeants du Congrès du travail du Canada et des chefs du Parti social démocratique du Canada (CCF) en vue de former un nouveau parti politique.

Tout d'abond le Congrès du travail du Canada (CTC), lors de son congrès de Winnipeg en avril 1958, a adopté une résolution proposant la formation «d'un mouvement politique populaire à cadres très larges groupant le PSD (CCF), le mouvement syndical, les associations agricoles, les professionnels et les citoyens d'esprit libéral. Voici le texte de cette résolution:

«Le présent congrès est convaincu de l'impérieuse nécessité à l'heure actuelle de créer une force nouvelle et efficace sur la scène politique canadienne, une force qui se fondera sur les besoins des travailleurs, des agriculteurs et des autres groupes populaires et qui sera financée et contrôlée par le peuple et ses organismes.

* Au cours des vingt-cinq dernières années, le PSD a grandement contribuée au bien commun du peuple canadien par son action tant au Parlement qu'en dehors du Parlement. Le mouvement syndical est conscient de cet apport et reconnaît que, dans la mesure de ses modestes moyens, le PSD continue de lutter en faveur des principes de justice sociale, de sécurité et de liberté, au triomphe desquels s'est également voué le CTC.

«L'heure est venue d'opérer un regroupement fondamental des forces politiques au Canada. On sent le besoin d'un vaste mouvement populaire, englobant le PSD, les syndicats ouvriers, les associations agricoles, les professionnels et les citoyens d'esprit libéral, en un mot tous ceux qui aspirent à une réforme et à une reconstruction sociales en profondeur, réalisées dans le cadre de notre système parlementaire de gouvernement. Cet instrument politique basé sur une participation populaire aussi large que possible devrait permettre au mouvement syndical et aux autres associations de même caractère, en collaboration avec le PSD, de contribuer directement à l'instauration d'un tel organisme, à l'élaboration de sa structure organique, à la formulation de ses principes de base et de son programme, ainsi qu'à son financement et au choix de ses candidats aux fonctions publiques.

«Il sera profitable d'examiner l'histoire et la structure des partis travaillistes et sociaux-démocrates en d'autres pays afin de s'inspirer et de tirer parti de leur expérience; mais il ne faudra jamais perdre de vue que, pour être efficace au Canada, un organisme politique doit être essentiellement canadien dans son caractère et dans sa structure.

«En lançant l'idée d'un nouveau parti politique et en participant à sa création, le mouvement syndical souligne le fait que non seule- 
ment il n'a pas l'intention de dominer un tel mouvement, mais qu'au contraire il souhaite vivement la plus vaste adhésion possible de tous les individus et groupements qui se préoccupent sincèrement d'une réforme en profondeur de notre société et qui la croient réalisable par des méthodes de planification démocratique.

Conséquemment, le présent congrès donne mandat au Conseil exécutif d'entreprendre immédiatement les démarches nécessaires, en engageant des pourparlers avec le PSD, les associations agricoles et les autres individus et groupements intéressés en vue de formuler des projets de constitution et de programme à l'intention de cet instrument politique du peuple canadien; le Conseil devra faire rapport du résultat de ses travaux et soumettre les dits projets aux délégués au prochain congrès général du CTC.

« Dans l'intervalle, le présent congrès réaffirme le principe posé dans la résolution politique adoptée lors du congrès de fondation du CTC en ces termes:

«(Le présent congrès) invite instamment tous les syndicats affiliés ainsi que les fédérations et conseils; a) à prendre un vif intérêt aux affaires politiques, b) à poursuivre les programmes d'éducation et d'action politiques dans lesquels ils sont déjà engagés, et c) à entreprendre résolument toute nouvelle activité susceptible de contribuer efficacement à la réalisation des objectifs fondamentaux du CTC;

« Et il presse d'autre part le Service d'éducation politique d'accorder toute l'aide possible aux syndicats affiliés, aux fédérations et aux conseils du travail dans la mise en oeuvre de leurs: programmes d'éducation et d'action politiques ».

De son côté, le Parti social-démocratique du Canada (CCF), lors: de son congrès national de juillet 1958, tenu à Montréal, a accueilli la résolution adoptée par le CTC comme étant « une étape décisive de notre histoire politique » et s'est déclaré prêt à collaborer pleinement avec le CTC et les autres individus et groupements intéressés afin de réaliser les objectifs définis dans la résolution.

Voici également le texte de la résolution du Parti social-démocratique:

«Le présent Congrès national du PSD réaffirme sa conviction que le bien-être du pays et du peuple canadien repose sur l'élargissement et la prompte victoire d'un mouvement politique groupant tous les secteurs de la population. En tant que sociaux-démocrates, nous croyons qu'un tel mouvement devra continuer à s'inspirer des principes de la planification sociale démocratique et préconiser les formes les plus amples de sécurité sociale et de liberté individuelle. Il devra demeu- 
rer inébranlable dans sa détermination d'introduire partout où ces mesures seront indiquées, le contrôle et la propriété publics pour remplacer la présente domination monopolistique de notre économie et de toute notre société par les grandes entreprises.

«Un tel mouvement devra se consacrer à la tâche de reconstruire démocratiquement notre société pour en arriver à ce que la coopération remplace l'âpreté au gain, pour que le développement ordonné remplace l'exploitation de l'homme par l'homme, pour que l'union entre fermiers et ouvriers, entre l'est et l'ouest, entre les Canadiens de langue française et les Canadiens de langue anglaise remplace les sentiments de discorde et les sources de conflit. Son objectif doit être de construire notre société sur les bases morales de la justice sociale et de la dignité humaine».

Pour ces raisons, le congrès du PSD reçoit d'une façon bienveillante la résolution adoptée par le Congrès du travail du Canada à sa convention de Winnipeg en avril 1958 en vue de fonder un tel mouvement politique, de concert avec le PSD, les organisations agricoles et les autres groupes et individus prêts à marcher vers les mêmes objectifs. "Lors de son congrès de fondation en 1956, le CTC a adopté un programme politique que le parti CCF, quelques mois plus tard, endossait avec empressement, ce qui établissait une fois de plus l'identité du programme du parti CCF avec les objectifs sociaux du travail organisé tout comme les objectifs du CCF sont identiques à ceux des organisations agricoles et d'autres groupes de la société. Depuis sa fondation d'ailleurs, le parti CCF a toujours fait appel au travail organisé et au mouvement agricole pour qu'ils se joignent à lui afin de bâtir un mouvement politique populaire qui soit fort et vraiment représentatif de toutes les classes de la société. La résolution du CTC marque une étape dans l'histoire politique de notre pays et offre l'occasion de faire de réels progrès.

«C'est pourquoi le congrès du PSD autorise son Conseil national et son exécutif national à rencontrer les dirigeants du Congrès du travail du Canada, ceux de la Confédération des travailleurs catholiques du Canada, les organisations agricoles et les autres groupes intéressés et lui demande de présenter un rapport de ces rencontres au prochain congrès national du parti ou lors d'un congrès spécial à cette fin. De plus, le congrès du PSD autorise son Conseil national et son Exécutif à entreprendre toutes les consultations et discussions nécessaires avec les membres et clubs du parti et demande que tout projet de constitution du nouveau parti soit soumis aux clubs et associations du parti pour discussions et recommandations avant d'être transmis au prochain congrès et que toute proposition, concernant le nouveau parti, qui devra être soumise au Congrès du PSD soit transmise aux clubs et associations CCF au moins deux mois avant la prochaine convention ». 
UN COMTTÉ CONJOINT

Après cette manifestation du désir de collaborer des deux groupes, un comité conjoint de dix représentants du CTC et de dix délégués du PSD fut formé. Dès sa première séance, le comité désigna M. Stanley Knowles comme président et M. Carl Hamilton comme secrétaire.

Le travail de ce comité consista tout d'abord à rechercher l'appui d'autres groupes et associations comme le mentionnaient les résolutions adoptées séparément. On forme un sous-comité qui fut chargé de rencontrer les représentants de la Confédération des travailleurs catholiques du Canada et les groupements agricoles. Les membres du comité avaient aussi l'autorisation d'entrer en contact, individuellement, avec toute personne d'esprit libéral.

Au cours de réunions subséquentes, le comité conjoint dut clarifier certaines situations. Tout d'abord, le CTC dut établir ses positions à l'égard de certains groupes qui, à l'intérieur de ses rangs, étaient opposés à la participation du mouvement syndical à la formation d'un nouveau parti. L'une des résolutions adoptées par le comité conjoint spécifie que le comité est d'accord avec la politique du CTC de «respecter les droits de toute union affiliée, en particulier des unions d'employés du gouvernement fédéral, des employés provinciaux et fédéraux, de demeurer indépendantes de tout parti politique».

Une autre résolution adoptée par le comité en réponse à certaines propositions faite par le Parti progressiste-ouvrier (communiste) met également les choses au point.

Cette brochure intitulée «Un nouveau parti politique pour le $\mathrm{Ca}$ nada » et imprimée en français et en anglais, traitait des objectifs du parti, des formules d'adhésion à l'usage des membres individuels et des groupes comme les syndicats, les coopératives et autres, de la structure du nouveau parti. Elle contenait également certains documents de base comme les deux résolutions adoptées par le PSD et le CTC en vue de former un nouveau parti politique et finalement elle contenait un tableau comparatif des anticles des programmes politiques du Congrès du travail du Canada (CTC) du Parti social démocratique (CCF), de la Fédération canádienne de lagriculture (FCA) et du Conseil interprovincial des syndicats agricoles.

Ce tableau mettait en évidence et parallèlement les positions de chacun de ces organismes sur la propriété publique, les investissements publics, les ressources naturelles, l'automation, la politique fiscale, les coopératives, l'assurance-santé, l'assurance-chômage, les pensions et allocations diverses, la politique ouvrière, la politique agricole, l'éducation, les affaires internationales, le commerce extérieur, etc.

Un sous-comité fut ensuite chargé de promouvoir l'organisation de cercles d'études, de seminars dans toutes les parties du pays afin 
de sonder l'opinion des syndiqués, des sociaux démocrates et des autres groupes intéressés.

C'est le faisceau de tous ces renseignements, de toutes ces informations recueillis à travers le pays, de ces échanges de vue que les: 300 délégués au seminar national de Winnipeg ont analysé, étudié, comparé afin de voir s'il y avait moyen de trouver une formule d'entente entre les intéressés.

LES DOCUMENTS DE TRAVAIL

Comme instruments de travail, les délégués disposaient des documents suivants:

1) un rapport des fédérations provinciales du parti CCF et des: fédérations de travailleurs affiliées au Congrès du travail du Canada;

2) un rapport des 25 seminars, écoles, cercles d'études et forums: organisés dans toutes les provinces canadiennes tant sous l'égide du CTC que du PSD;

3) un rapport d'un sous-comité sur la constitution du nouveau parti;

4) un rapport du sous-comité sur la politique du nouveau parti.

\section{LES RAPPORTS PROVINCIAUX}

Les rencontre faites à l'échelon provincial, tant du côté du CTC que du PSD, ont été à peu près unanimes à recommander la formation d'une nouveau parti politique à base largement démocratique.

Bornons-nous à signaler le rapport fourni par les représentants: du Québec et celui des représentants du CCF de la Saskatchewan.

Le comité conjoint formé dans le Québec pour étudier lopinion des syndiqués et des autres groupements en vue de fonder un nouveau parti politique, a mis l'aocent, dans son rapport, sur la nécessité de tenir compte des besoins particuliers des provinces et particulièrement de la province de Québec.

Ce comité conjoint a siégé à quatre reprises au cours de l'année et était composé de MM. Roger Provost, Edouard Larose, Eucher Corbeil, Roméo Girand et Pat Burke, de la Fédération des travailleurs du Québec et de 4 membres de son comité d'action et d'éducation politiques: Ken Dewitt, L. H. Lorrain, Jean Philip et Jacques V. Morin. Le PSD était représenté par Michel Chartrand, Emile Boudreau, Cyrille H. Durocher, Michel Forest, Gabriel Gagnon et Mme Thérèse Casgrain. Le comité a cherché à éviter l'erreur du PSD où l'absence 
de représentants authentiques du Québec et des Canadiens français l'a privé de l'appui des masses populaires du Québec et a été l'une de ses faiblesses sur le plan national.

Plusieurs membres du comité sont d'opinion que le nouveau parti devrait mettre l'accent sur la fondation de partis provinciaux. Ceci est particulièrement vrai pour la province de Québec en ce qui a trait à l'éducation, aux ressources naturelles et à la législation ouvrière. D'autres, par contre, favorisent un parti national tout en admettant que les structures et le programme de ce parti devraient tenir compte des besoins et des aspirations du Québec.

Tous les membres du comité sont d'accord que pour lancer un nouveau parti dans la province de Québec il faut que ce parti puisse s'assurer les services d'un organisateur dont l'unique tâche serait de fonder des sections, rencontrer les organisations syndicales qui sont susceptibles de s'y affilier et faire un travail d'éducation.

Le comité a fait état d'une rencontre avec les dirigeants du Rassemblement qui auraient montré un intérêt à la fondation d'un nouveau parti politique.

Par ailleurs MM. Michel Chartrand et Roger Provost ont eu des rencontres officieuses avec des dirigeants de l'Union catholique des cultivateurs qui sont intéressés dans cette nouvelle formation, mais qui ne veulent pas impliquer leur organisme.

Le comité suggère donc la tenue de rencontres régionales avec les dirigeants régionaux de l'UCC et de la Confédération des travailleurs catholiques du Canada et d'autres organisations affiliées.

\section{Hésitation en Saskatchewan}

Le rapport soumis par le Conseil provincial du parti PSD (CCF) de la Saskatchewan qui détient le pouvoir depuis 15 ans a jeté un peu de froid sur l'enthousiasme des délégués.

Tout d'abord le comité provincial avait élaboré un plan pour permettre aux organisations de comtés de discuter la formation d'un nouveau parti en leur faisant parvenir un document qui groupait les quatre questions suivantes:

1) Le nouveau parti politique aura-t-il des ressources financières plus grandes et le personnel nécessaire pour bâtir une organisation efficace dans toutes les parties du pays? 2) y a-t-il danger que le mouvement ouvrier domine le nouveau parti? 3) peut-on combiner l'adhésion d'organisations affiliées avec celle d'individus dans un parti démocratique? 4) y a-t-il différence entre la politique du PSD et du CTC? 
On a envoyé une copie de ces questions à tous les secrétaires des organisations de comtés leur demandant de les discuter lors de leurs congrès annuels. On leur a également suggéré de rédiger un projet de résolution à soumettre au prochain congrès général du parti à Saskatoon.

Plusieurs comtés ont soumis des résolutions approuvant les études conjointes des comités mixtes PSD-CTC. La majorité des résolutions soumises par les organisations de comtés s'opposaient à la création d'un nouveau parti politique.

On traite de la façon suivante la résolution concernant le nouveau parti, au congrès provincial. Chacun des cinq panels au congrès ont discuté les quatre questions inscrites plus haut. Les cinq présidents des panels ont formé un comité qui a soumis la résolution suivante:

1-Il est proposé que ce congrès appuie les discussions préliminaires du comité mixte PSD-CTC en vue de la formation d'un nouveau parti;

2-que le Conseil provincial encourage tous les clubs PSD, les organisations de comtés et de districts à discuter de la formation d'un tel parti, et qu'il prenne les mesures appropriées afin de fournir les documents nécessaires à une discussion officieuse de cette proposition;

3-qu'aucune décision concernant la formation d'un tel parti ne soit prise tant que la proposition n'aura pas été discutée aux congrès de comtés et au congrès provincial du PSD de Saskatchewan.

Attrtude de Douglas

Adressant la parole aux délégués réunis à Winnipeg, au cours de la dernière journée du séminar, le premier ministre de la Saskatchewan, M. T.C. Douglas, a lancé un appel à l'unité des travailleurs et des cultivateurs.

«La Saskatchewan, a-t-il dit, a prouvé que les ouvriers et les fermiers peuvent travailler la main dans la main. Bien que les cultivateurs aient constitué la majorité dans le premier gouvernement CCF, ils ont adopté la meilleure législation ouvrière sur le continent et les deux groupes ont toujours travaillé en parfaite harmonie, a-t-il dit.

«La propagande des compagnies cherche à convaincre les ouvriers que leurs intérêts diffèrent de ceux des cultivateurs. D'autre part les fermiers ont souvent tendance à blâmer les ouvriers. Je leur demande s'ils vivaient mieux quand le salaire minimum des travailleurs était de $\$ 9.00$ par semaine. 
«La hausse des salaires a permis de doubler la consommation de la viande depuis 1937. Le jour où nous retournerons à l'ère des salaires de famine et au ohômage, les prix de la ferme baisseront.

«Par ailleurs, dit-il, le revenu du travailleur industriel dépend en large mesure du pouvoir d'achat des fermiers ». Il a fait état des difficultés que rencontrent les fermiers qui doivent vendre leurs produits sur le marché libre tandis que les tarifs et les cartes protègent les manufacturiers.

«Il a réclamé la parité des prix pour l'agriculture. Cela coûterait approximativement $\$ 300$ millions par année alors que la politique tarifaire du gouvernement coûte plus d'un milliard aux consommateurs par année.

«La prospérité est indivisible tout comme la paix», a-t-il dit.

En concluant ses remarques, M. Douglas a ajouté que «le nouveau parti n'aura peut-être pas l'appui de la masse des fermiers à ses débuts mais ils y viendront. Nous pourrons dire un jour qu'aujourd'hui nous avons allumé une flamme qui ne s'éteindra pas ».

Ces paroles soulevèrent l'enthousiasme des délégués car on avait maintes fois souligné la crainte des partisans du parti CCF de la Saskatchewan d'être noyés dans ce nouveau parti. Ils craignent, par exemple, que le changement de nom du parti, une nouvelle politique, leur nuisent sur le plan provincial en Saskatchewan.

\section{LA POLITIQUE DU NOUVEAU PARTI}

Le programme du nouveau parti avait été élaboré par un souscomité qui a soumis un document de base de 32 pages aux délégués. Le document traitait des sujets suivants:

L'agriculture, le contrôle et la gestion de l'économie, le logement, l'assistance économique aux autres pays, le travail, les nationalisations, la sécurité sociale, la taxation et l'éducation.

Ce document analysait les positions du parti CCF sur ces problèmes et suggérait de les compléter afin de les rendre plus adéquates aux besoins actuels.

La discussion sur ces sujets s'est faite à huis-clos par quatre commissions qui avaient deux sujets à explorer. Les rapports de ces conclusions ont été ensuite soumises aux délégués réunis en assemblée plénière.

La commission sur les problèmes du travail a porté son attention sur le problème de l'automation, ainsi que le suggérait le document de travail. 
Au sujet de l'automation, de ses effets sur la main d'oeuvre et sur l'économie en général, le comité, sous la présidence de Grace McInnis, présidente du parti CCF de la Colombie Britannique, s'est dit d'avis que tout programme économique satisfaisant devait tenir compte de la planification. "Le syndicalisme seul ne pourra suffire à la tâche. Nous devrons prouver que la politique peut travailler dans les intérêts du peuple».

Le comité croit que l'automation permettra d'accorder plus de loisirs à la population et facilitera l'épanouissement de l'éducation.

Le nouveau parti devra s'employer à réaliser le plein emploi, améliorer la loi de l'assurance-chômage, garantir à tous le droit à la négociation collective.

\section{Les nationalisations}

Le document de base sur les nationalisations soulignait qu'il n'y a « rien de bon ou de mauvais en soi dans les nationalisations et qu'on ne pouvait les juger qu'en fonction d'industries ou d'entreprises particulières» selon certains critères: bénéfice net au point de vue bienêtre, la meilleure formule après avoir essayé les autres, une distribution plus large des revenus et une production accrue.

M. Harold Winch, MP a présenté le rapport de cette commission en énonçant les suggestions suivantes exprimées par son groupe: 1) un programme d'éducation pour informer le public des avantages de la propriété publique; 2) une étude de ce que le nouveau parti voudrait nationaliser dès son premier terme d'office; 3) un reproche au gouvernement actuel qui sabote les entreprises publiques comme Air-Canada et Radio-Canada.

\section{Sécurité sociale}

Le trésorier national du parti CCF, Andrew Brewin, a présenté le rapport du groupe ayant étudié les problèmes de la sécurité sociale et de la taxation. Le groupe considère que les mesures de sécurité sociale doivent s'échelonner selon un ondre de priorité: 1) la protection de la santé par le moyen d'un plan d'assurance-santé national; 2) la protection contre le chốmage; 3 ) un plan contributoire de retraite avec «pension mobile»; 4) l'ajustement des prestations diverses selon l'indice du coût de la vie.

Le groupe croit qu'un bon système de taxation doit servir d'instrument de justice sociale et de planification économique. Nous devrons reviser le système fiscal afin que personne ne puisse échapper à ses obligations de «façon légale ou illégale», a-t-on dit. 
On a également déclaré que le Canada étant un pays fédéral où les provinces et les municipalités ont des devoirs et des droits, il fallait reconnaître le rôle positif que jouent ces gouvernements et leur donner les moyens financiers nécessaires pour faire face à leurs responsabilités.

\section{Les relations économiques}

La commission présidée par le professeur Frank Scott a porté son attention sur les relations économiques du Canada avec les autres pays.

Concernant les investissements américains, on a répondu qu'il s'agissait de savoir s'ils favorisaient une politique sociale. C'est ce principe, a-t-on dit, que le nouveau parti devra appliquer, quelle que soit la provenance des capitaux investis.

Cependant le comité a constaté que les investissements américains au Canada permettent aux Etats-Unis de contrôler de vastes secteurs de notre économie. Le comité croit que la recommandation de la Commission royale Gordon d'obliger les entreprises américaines au Canada à utiliser un certain pourcentage de capitaux canadiens n'est pas suffisante. Le comité a également exprimé qu'en certains cas, la propriété publique se justifierait pour développer certaines entreprises canadiennes qui sont obligées à recourir à des capitaux américains. On a cité le cas du pipeline transcanadien.

On a également souligné que des emprunts aux Etats-Unis ou ailleurs permettraient au Canada de conserver la main mise sur notre économie.

\section{L'agriculture}

Concernant les problèmes de l'agriculture étudiés par la commission présidée par M. Hazen Argue, les délégués ont découvert que les agriculteurs devraient se tracer des objectifs précis tout comme les organisations syndicales.

Le comité s'est déclaré d'accord sur une politique de parité des prix. Ces derniers devraient s'ajuster selon le coût de la production et des investisements des agriculteurs. Dans le comité, les syndicalistes ont déclaré être favorables à la politique agricole préconisée par les fermiers.

Au sujet de l'intégration verticale, les représentants sont opposés au système actuel d'intégration mais ils favorisent une intégration par l'intermédiaire de coopératives au bénéfice des producteurs et des consommateurs. 


\section{L'éducation}

Les problèmes de l'éducation devront être résolus par les sections provinciales du nouveau parti. Les délégués toutefois ont affirmé qu'il faut faire disparaître tous les obstacles économiques et sociaux qui entravent le développement intellectuel. Le nouveau parti favorisera également «la juridiction exclusive des provinces sur l'école et l'éducation ». Les délégués se sont prononcés en faveur de l'instruction gratuite à tous les niveaux, de l'aide aux institutions par les gouvernements appropriés, et de meilleures conditions de travail pour les professeurs.

\section{Le rôle du Comité conjoint}

Le Comité conjoint PSD-CTC qui avait été chargé de préparer une ébauche de programme du nouveau parti a reçu ces rapports pour information et il sera maintenant en mesure de tracer le programme du nouveau parti.

Ce programme, la constitution du parti, les modalités concernant l'admission des membres, seront soumises aux congrès respectifs du Parti social démocratique qui aura lieu l'an prochain de même qu'au congrès du CTC qui se réunira à Montréal en avril 1960.

Si tout se passe comme prévu, les deux groupes se réuniront de nouveau, après ces congrès, pour fonder véritablement le nouveau parti, définir son programme et lui donner une constitution appropriée.

Pour conclure, je cite de nouveau, M. Stanley Knowles adressant la parole à l'issue des séances d'études:

* Le Comité national conjoint, a-t-il dit, va maintenant organiser des journées d'étude, des seminars sur la constitution et le programme du nouveau parti à travers le pays.

"Le Comité s'est déjà donné un sous-comité chargé de rédiger une ébauche de cette constitution et ce programme. Il travaillera, de concert avec le Comité conjoint, à faire une nouvelle rédaction qui tiendra compte des points de vue échangés au cours de ce seminar.

«En quittant ce seminar, nous allons de l'avant et nous approchons davantage du jour de fondation du nouveau parti ».

LA représentation du Qú́Bec

Il y avait plus de 300 délégués au seminar de Winnipeg. 119 de ces délégués représentaient des organisations du Parti social-démocratique (CCF); 116 représentaient des organisations syndicales, 33 représentaient des fermiers et il y avait 36 participants provenant de milieux divers, dont des avocats, des universitaires, des journalistes, etc. 
Le Québec avait délégué une vingtaine de représentants. Parmi ces derniers remarquons M. Gérard Picand, membre du Conseil national du PSD, Mme Thérèse Casgrain, également membre du Conseil national; M. Roger Provost, président de la Fédération des travailleurs du Québec; Philippe Vaillancourt, directeur régional du Service d'Education du CTC, pour la province de Québec; Yvan Legault, secrétaireexécutif de la FTQ; Emile Boudreau, président provincial du PSD; Edouard Larose, Eucher Corbeil, Roméo Girard, Pat Burke, Jean Philip, Jacques V. Morin, tous membres d'organisations syndicales affiliées à la FTQ et au CTC. La CTCC comme telle n'avait aucun représentant. Me Pierre Vadeboncoeur, conseiller technique de la CTCC, assistait à titre de membre du conseil provincial du PSD.

A l'issue du seminar, les délégués du Québec ont tenu une conférence de presse pour affirmer qu'ils vont veiller à ce que le nouveau parti trouve une solution véritable et satisfaisante au problème des relations fédérales-provinciales.

Mme Thérèse Casgrain, MM. Roger Provost et Gérard Picard, parlant au nom de la délégation québecoise ont déclaré que les autres provinces sont aussi désireuses de régler le problème « autrement que par des expédients politiques qui empoisonnent depuis trop longtemps la nation canadienne ».

Les trois porte-parole ont affirmé que « les vieux partis ont intérêt à ne pas régler la querelle des relations fédérales-provinciales parce que ça leur permet d'exploiter électoralement leur chicane avec Ottawa pour escamoter les véritables problèmes intéressant le bien-être de la population ».

Ils ont cité en exemple le cas du Québec qui fait ses élections avec l'autonomie sans jamais proposer de solution au problème.

« Le principe qui doit présider à la solution de l'imbroglio des relations fédérales-provinciales, ont-ils dit, c'est une fiscalité qui donnerait à Ottawa et à chacune des provinces des revenus suffisants pour s'acquitter de leurs devoirs constitutionnels. Cependant seul un parti populaire peut régler vraiment le problème, car les vieux partis ne peuvent tirer des revenus supplémentaires de nouvelles sources de taxation, comme les gains de capital. Ils perdraient alors de généreuses contributions des grandes compagnies à leur caisse électorale.

\section{Allocution de M. Claude Jodoin}

Le président du Congrès du travail du Canada, M. Claude Jodoin a adressé la parole à deux reprises aux délégués. Dès l'ouverture du seminar, il a prononcé une allocution en affirmant que le seminar de Winnipeg découlait des instructions données par le CTC à son exécutif de travailler à la formation d'un nouveau parti politique. 
«Le CTC, a-t-il dit, croit au droit des Canadiens de choisir entre deux partis dont l'un serait de droite, l'autre de gauche en excluant toutefois l'extrême droite fascite et l'extrême gauche communiste.

Rappelant les lois antisyndicales de Terre-Neuve et de la ColombieBritannique, il a accusé les libéraux et les conservateurs de ne pas avoir eu le courage de définir leur attitude à ce sujet.

Il a dit que toutes les classes de la société faisaient de l'action politique: avocats, notaires, médecins, fermiers, etc.

«Ai-je besoin de mentionner les employeurs, a-t-il demandé? Il est connu que les vieux partis obtiennent l'aide financière des employeurs, des compagnies et des grosses entreprises. Est-ce un mal? N'ont-ils pas le droit d'appuyer leurs amis? Certainement. Mais les ouvriers de ce pays n'ont-ils pas aussi ce droit? N'ont-ils pas un droit égal de supporter un parti politique de leur choix »?

M. Jodoin a ensuite exposé la position du Congrès du travail en ces termes:

«Le Congrès du travail du Canada et ses syndicats affiliés ne veulent d'aucune manière contrôler le nouveau parti ni aucun parti politique. Nous, du CTC, nous voulons coopérer avec les autres classes de la société pour construire un Canada meilleur.

«Nous, du Congrès du travail du Canada, nos membres, nos familles, nous représentons $1 / 5$ de la population canadienne. Nous sommes le Canada en action ».

Parlant du rôle du CTC dans le nouveau parti, il a dit ce qui suit:

«Les décisions finales devront être prises par le congrès biennal du CTC, qui constitue l'autorité suprême du mouvement: je crois cependant que la centrale elle-même, soit le CTC, ne devrait pas être affiliée à un parti politique ».

Il a cité l'exemple du TUC anglais qui n'est pas affilié au parti travailliste.

«Le Congrès du travail du Canada, dit-il, constitue une organisation centrale composée de plusieurs syndicats qui voudront exercer leurs propres droits démocratiques et s'abstenir de toute forme d'action politique.

«C'est un droit que nous voudrons protéger et, à cause de cela, il convient que le CTC lui-même s'abstienne de toute forme d'action politique ».

Il a aussi déclaré que le CTC avait des principes, une politique à sauvegarder et qu'il devrait rester libre d'approuver ou de critiquer toute action gouvernementale prise par tout parti politique ». 
Certains ont interprété cette déclaration comme une reculade de la part du Congrès du travail du Canada. Les délégués à la convention annuelle du CTC en 1958 n'avaient-ils pas donné instruction à l'Exécutif du CTC de travailler à la formation d'un nouveau parti politique? Le CTC reculait-il? Claude Jodoin lui-même se dérobait-il?

Certains journalistes ont interprété les remarques de M. Jodoin de cette façon. Ils ont commenté cette allocution en mettant dans la bouche de M. Jodoin des mots qu'il n'avait pas dits.

La Presse Canadienne qui avait un représentant à Winnipeg est grandement responsable des conclusions qui ont été tirées du discours de M. Jodoin. Le soir même, soit vendredi, le 28 août, Radio-Canada diffusait à travers le pays une nouvelle tendancieuse, sur la foi de la Presse Canadienne.

Le lendemain, M. Jodoin a été obligé de rétablir les faits, d'apporter des précisions en ces termes:

«Par suite de certains compte-rendus de journaux qui ont mal interprété les positions du CTC et mes opinions personnelles, je suis obligé de faire la mise au point suivante, a-t-il dit:

«La décision prise par le CTC, lors de son congrès de 1958, d'amorcer des discussions avec le PSD en vue de former un nouveau parti politique groupant le PSD, le travail organisé, les organisations de fermiers et tous les esprits libéraux furent pratiquement unanime et avaient mon entier appui.

« Depuis cette décision, le CTC a joué un rôle actif. Selon le mandat que nous avions reçu, nous avons accordé la priorité à la tâche de déblayer le terrain en vue de la formation d'un nouveau parti. Ceci, encore, a reçu de ma part un appui sans réserve.

\& De plus, je suis persuadé que la très grand majorité des membres des unions affiliées au CTC ainsi que les unions elles-mêmes appuient fermement et appuieront encore plus fermement l'idée de la formation d'un nouveau parti.

«Dans les déclarations que j’ai faites, j’ai mis l'accent sur deux choses: le travail organisé veut collaborer avec les autres classes de la société et ne cherche pas à dominer le nouveau parti; 'jai ensuite déclaré que le Congrès du travail du Canada lui-même, comme centrale, ne devrait pas s'affilier au nouveau parti mais que les unions qui sont membres du CTC devraient le faire.

«Ceci, dit-il, a été interprété dans les journaux comme une reculade du Congrès. Cette interprétation est tout à fait fausse.

« Notre position n'est pas nouvelle. Elle a été clairement expliquée dans la brochure publiée par le Comité conjoint PSD-CTC. 
«Le Congrès du travail du Canada et moi-même, à titre de président, nous croyons que le besoin d'un nouveau parti se fait plus grandement sentir aujourd'hui qu'il y a un an. Les récentes lois antiouvrières adoptées depuis nous ont fait prendre plus fermement conscience que ni le parti libéral, ni le parti conservateur ne désirent nous donner justice et protéger nos droits.

«Toutefois, le Congrès du travail du Canada qui est composé de plusieurs organisations affiliées, mais qui sont autonomes, doit laisser à chacune des unions la complète liberté de faire leur propre choix au sujet de l'affiliation et le CTC doit également rester lui-même libre d'exercer ses fonctions représentatives auprès des gouvernements.

« Cela n'infirme pas l'appui que nous accordons au nouveau parti. C'est la politique suivie d'ailleurs par le TUC en Grande-Bretagne à l'égard du parti travailliste. Toutefois, le TUC ne dissimule pas son appui au parti travailliste et le Congrès du travail du Canada et ses unions affiliées accorderont leur entier appui au nouveau parti qu'ils sont en train de former $»$.

\section{Allocution de David Lewis}

Le président du parti CCF, David Lewis, adressant la parole lors de la séance d'ouverture, a déclaré que la procédure démocratique ne permet pas toujours des développements rapides et spectaculaires.

«Nous discutons l'idée de former un nouveau parti depuis un an et demi. Nous nous sommes rencontrés, nous avons organisé des journées d'études à travers le pays. Nous continuerons ainsi jusqu'après les congrès du PSD et du CTC en 1960. Cela signifie une autre année de discussions, de rencontres, d'études.

«Mais quand le nouveau parti sera officiellement fondé, a-t-il ajouté, quand sa politique, son programme, sa constitution seront proclamés, il sera l'oeuvre de milliers et de milliers de Canadiens qui auront réfléchi, qui auront discuté, qui auront travaillé ensemble à donner à leur pays un instrument démocratique, forgé démocratiquement et contrôlé démocratiquement.

M. Lewis a ensuite lancé un appel aux organisations syndicales et aux fermiers pour qu'ils s'unissent au parti CCF pour fonder un grand parti canadien qui sera dévoué à la défense de leurs intérêts.

Après avoir rappelé que le parti CCF a été pendant des années « la conscience de la politique canadienne» et que malgré son peu de succès, il avait résisté au balayage des conservateurs sur le plan national tandis que le parti détenait le pouvoir depuis 15 ans en Saskatchewan, il a fait allusion aux attaques qu'on lance actuellement contre les organisations syndicales que l'on veut affaiblir et détruire. 
Il a dit que l'histoire se répétait à ce sujet et que le mouvement syndical anglais avait traversé les mêmes épreuves avant de se lancer dans l'action politique.

Il a ensuite mis les fermiers en garde contre la propagande faite contre le mouvement ouvrier qu'on accuse d'être responsable des hausses de prix de l'industrie.

«Le fait brutal, a-t-il dit, c'est que les hausses de salaires n'ont que peu à voir, sinon rien, avec les augmentations de prix dans l'entreprise monopolistique moderne. Dans les industries de base comme l'acier et l'automobile, les augmentations de prix ont été plus fréquentes et plusieurs fois plus élevées que les hausses de salaires.

«L'inflation que nous venons de connaître ne s'explique pas par la théorie classique: trop d'argent en face de peu de produits. Au contraire, a-t-il dit, aujourd'hui nous assistons à des hausses de prix au moment où la demande diminue. La vérité, c'est que les prix dans lindustrie moderne sont calculés en fonction du profit à réaliser. L'inflation actuelle est en grande partie créée délibérément par les entreprises qui manipulent les prix, qui aocumulent d'immenses réserves à même les profits sans précédent qu'elles réalisent ».

Il a ajouté que bientôt la lumière se fera au sujet des problèmes économiques et du rôle des organisations syndicales, et que les fermiers et les autres classes de la société seront prémunis contre les mensonges que l'on colporte à leur sujet.

Il a avoué que le parti CCF n'avait pas d'assises populaires assez larges pour être un parti populaire.

«Nous savons, dit-il, qu'il est essentiel de mobiliser le support du travail organisé et des fermiers pour asseoir solidement les bases d'un grand parti. C'est lunique raison pour laquelle le CCF a endossé aussi chaleureusement la résolution du Congrès du travail du Canada. Car personne dans le CCF n'a été intéressé ou n'est intéressé au pouvoir comme tel. Personne n'est assoiffé de prestige. Nous croyons seulement en des principes et en un idéal de justice sociale. Le parti CCF prend une part active à la formation de ce nouveau parti parce que nous sommes convaincus que l'étape que nous franchissons actuellement enrichira les principes d'un mouvement politique populaire et hâtera l'avènement de notre idéal commun ».

\section{Le respect des charges de famille dans la taxation et la sécurité sociale au Canada}

\section{Cllaude Morno}

Actuellement, les dépenses qu'effectuent les trois niveaux de gouvernement au Canada en vertu des divers programmes de maintien du revenu et de santé atteignent 8 à $9 \%$ du revenu national net et en- 